\title{
Supporting Information Local Density Fluctuations Predict Photoisomerization Quantum Yield of Azobenzene-Modified DNA
}

\author{
Addie Kingsland, Soumyadyuti Samai, Yunqi Yan, David S. Ginger, Lutz Maibaum \\ Department of Chemistry, University of Washington, Seattle, WA, USA
}

\section{METHODS}

\section{Materials}

Azobenzene modified and unmodified DNA sequences for matched, ssDNA, mm1A, mm1C, mm1T, PM-abasic, mmabasic, mm-double-abasic, ss-polyT (shown in Table 1) were synthesized and purified with HPLC by Integrated DNA Technology (IDT Inc., IA). Reagents including sodium chloride $(\mathrm{NaCl})$, disodium hydrogen phosphate $\left(\mathrm{Na}_{2} \mathrm{HPO}_{4}\right)$, potassium dihydrogen phosphate $\left(\mathrm{KH}_{2} \mathrm{PO}_{4}\right)$, and sodium azide $\left(\mathrm{NaN}_{3}\right)$ were purchased from Sigma Aldrich and used as received. Water used in the experiments were deionized to 18.2 $\mathrm{M} \Omega$ using the Millipore filtration system.

\section{Preparation of dsDNA solution for QY measurement}

Aliquots of a desired amount of lyophilized DNA were dissolved in water and the concentration was adjusted to $\sim 30 \mu \mathrm{M}$ in $10 \mathrm{mM}$ phosphate buffer containing $0.1 \mathrm{M} \mathrm{NaCl}$ and $0.05 \%$ sodium azide. The concentration of the DNA solution was determined from the absorbance at $260 \mathrm{~nm}$, measured with an Agilent $8453 \mathrm{UV}-\mathrm{V}$ is spectrometer. A slight excess amount of the complementary DNA sequence was added to the azobenzene modified DNA at room temperature and the mixture was annealed at $95^{\circ} \mathrm{C}$ for 5 minutes. It was then slowly cooled down to room temperature and stored at $4{ }^{\circ} \mathrm{C}$ before use. The final concentration of the dsDNA solution was about $15 \mu \mathrm{M}$.

\section{Quantum Yield Measurement}

For measuring the trans-to-cis photoisomerization quantum yield in different sequences, a DNA solution was irradiated with $330 \mathrm{~nm}$ UV light at $27^{\circ} \mathrm{C}$ and the absorbance of the solution at $330 \mathrm{~nm}$ was recorded every 1 minute for the first 20 minutes, every 2 minutes for the next 20 minutes, and then every 5 mintutes until 2 hours with an Agilent $8453 \mathrm{UV}-\mathrm{Vis}$ spectrometer. The temperature of the solution was maintained at $27^{\circ} \mathrm{C}$ using a calibrated temperature controller coupled with a home-made aluminum stage used as the cuvette holder. The solution was constantly stirred during the experiment. The intensity of the UV light was between 0.25 and $0.30 \mathrm{~mW} / \mathrm{cm}^{2}$, as measured by a calibrated $\mathrm{Si}$ photodiode for all the experiments.

\section{Quantum Yield Calculation}

We followed the procedure developed by Zimmerman et al. ${ }^{1}$ that we have previously adapted for our use. ${ }^{2}$ The following integrated rate expession is used to determine the quantum yield of azobenzene photoisomerization: ${ }^{1,3}$

$$
\mathrm{y}(t)=\left(\mathrm{y}_{0}-\mathrm{y}_{\infty}\right) \exp (-\mathrm{Ax}(t))+\mathrm{y}_{\infty} .
$$

Here $\mathrm{y}(t), \mathrm{y}_{0}$, and $\mathrm{y}_{\infty}$ are the fraction of cis-azobenzene at time $t$, time 0 , and in the photostationary state, respectively, and $\mathrm{x}(t)=\int_{0}^{t} \frac{\left(1-10^{-\mathrm{abs}(\mathrm{t} /)}\right)}{\mathrm{abs}(\mathrm{t} /)} \mathrm{dt}^{\prime}$ is the integrated photokinetic factor where $\operatorname{abs}\left(\mathrm{t}^{\prime}\right)$ denotes the absorbance of the solution at the irradiation wavelength $\lambda$ at time $t^{\prime}$. The prefactor $\mathrm{A}=\mathrm{I} \mathrm{l} \frac{\varphi_{\text {trans }} \varepsilon_{\text {trans }}}{\mathrm{V}_{\mathrm{y}_{\infty}}}$ additionally depends on the irradiation intensity I, the optical path length $1(1 \mathrm{~cm})$, the trans-to-cis photoisomerization quantum yield $\varphi_{\text {trans }}$, the total volume of the solution $\mathrm{V}$, and the extinction coefficient $\varepsilon_{\text {trans }}$ at irradiation wavelength $\lambda$, obtained from the literature. ${ }^{1}$ For our experiments, the excitation wavelength $\lambda$ is $330 \mathrm{~nm}$. The fraction of cis-azobenzene as a function of time, $\mathrm{y}(\mathrm{t})$, during UV irradiation was obtained from the absorbance at $330 \mathrm{~nm}$. The fraction of cis-azobenzene was plotted as a function of the integrated photokinetic factor and Igor Pro 6.3.6.4 was used to fit the experimental data (Supplementary Figure S1). The experimental quantum yield is then calculated from the values of $\mathrm{A}$ and $\mathrm{y}_{\infty}$ obtained from the fit.

The experiments were performed, following the method described above, on the sequences matched, PM-abasic, mm1T, mm1C, mm1A, mm-double-abasic and ssDNA. The quantum yield values obtained were slightly lower than the values reported in an earlier paper, ${ }^{2}$ which might be due to differences in the LED intensity measurement calibrations between the two experimental set ups. For sequence mm-abasic and sspolyT, the previously published values were normalized and recalibrated to be consistent with the experimental values obtained with the measurement protocol described herein.

\section{Force Field Parameterization}

Computer simulations were performed using the CHARMM27 molecular mechanics force field. ${ }^{4}$ This parameter set was used to describe all atoms with the exception of the central nitrogens forming the azo double bond. For these nitrogens, nonbonded interaction parameters were taken to be the same as standard CHARMM27 nitrogens. Bonded interactions were taken to be the same as those of the CHARMM27 aromatic atomtype $\mathrm{CN} 3$, with the exception of the $\mathrm{CCNN}$ and $\mathrm{CNNC}$ dihedral angles which were taken from calculations performed 
by McCullagh et. al. ${ }^{5}$ Because these two angles are coupled during azobenzene isomerization, we included a $\mathrm{CMAP}^{6}$ correction term to the trans dihedral angles which results in an additional energy minima at the calculated ground state geometry of the cis form. ${ }^{5}$ Partial charges for the azobenzene and linker atoms were generated using the $\mathrm{HF} / 6-31 \mathrm{G}^{*}$ electrostatic potential and fitted using the restrained electrostatic potential module in Amber. ${ }^{7-8}$

\section{Model Building}

Atomistic simulation models of B-DNA polymer containing twelve base pairs (table 1) were constructed using 3DNA software. ${ }^{9}$ DNA models were constructed using $3 \mathrm{DNA}^{9}$ and $\mathrm{VMD}^{10}$ was used to manually insert azobenzene at the correct position. Each system was minimized for 200 steps of steepest descent. The structures were solvated with explicit TIP3P water ${ }^{11}$ in a dodecahedral simulation box extending at least $1 \mathrm{~nm}$ beyond the DNA polymer in all directions. $22 \mathrm{Na}^{+}$counterions were randomly substituted for water molecules in order to neutralize the system. The solvent was minimized for 200 steps of steepest descent and then equilibrated for 100 ps. Each full system was then equilibrated for $1 \mathrm{~ns}$.

\section{Simulation Parameters}

Simulations were performed using the Gromacs 4.5.5 simulation suite. ${ }^{12}$ A 2 fs timestep was used and simulations were run for $100 \mathrm{~ns}$ each with trajectory coordinates saved every $10 \mathrm{ps}$. The system temperature of 300 Kelvin was maintained using stochastic velocity rescaling. ${ }^{13}$ The lengths of covalent bonds that include a hydrogen atom were constrained using the SHAKE algorithm. ${ }^{14}$ Long-range electrostatic interactions were treated with the Particle Mesh Ewald method ${ }^{15}$ with a non-bonded cutoff of $1.2 \mathrm{~nm}$.

\section{Occupation Number Calculation}

The number of atoms in the immediate vicinity of the azobenzene group was calculated for each simulation frame using the counting function

$$
N=\sum_{i \in A} \sum_{j \in B} \frac{1-\left[\left(r_{i j}-d_{0}\right) / r_{0}\right]^{6}}{1-\left[\left(r_{i j}-d_{0}\right) / r_{0}\right]^{12}}
$$

as implemented in the PLUMED 1.3.0 plugin. ${ }^{16}$ Here $\mathrm{A}$ is the set of atoms in the outer phenyl ring of azobenzene, $\mathrm{B}$ is the set of remaining atoms in the DNA, and $r_{i j}$ is the distance between atom $i$ and atom $j . d_{0}$ and $r_{0}$ are length parameters chosen to be $2.5 \AA$ and $0.5 \AA$, respectively. With these choices, the summand is an indicator function that is approximately equal to one if the distance falls within 2 to $3 \AA$ (Supplementary Figure S2). These parameters were optimized to maximize the correlation between experimentally determined quantum yield and the variance $\sigma^{2}$ of the occupation number (see below).

Statistics of the number of close contacts between the outer phenyl ring and the remaining DNA atoms were obtained by calculating the probability distribution $P(N)$, from which the free energy

$$
F(N)=-k_{B} T \log [P(N)]
$$

was calculated. Here, $k_{B}$ is the Boltzmann constant and $T$ is the temperature of the simulation. The variance of the occupation number was calculated as

$\sigma^{2}=\left\langle N^{2}\right\rangle-\langle N\rangle^{2}$, where the angular brackets denote the time average over the course of the simulation. Correlations between experimental and simulated data were quantified by a linear least-squares fit.

\section{Structure Calculations}

DNA structural parameters were calculated using $3 \mathrm{DNA}^{9}$ and analyzed using in-house computer code. Solvent accessible surface areas were calculated using $g$ sas from the Gromacs ${ }^{12}$ 4.5.5 simulation suite and analyzed using in-house computer code.

\section{REFERENCES}

1. Zimmerman, G.; Chow, L. Y.; Paik, U. J., The Photochemical Isomerization of Azobenzene. J Am Chem Soc 1958, 80, 3528-3531.

2. Yan, Y.; Wang, X.; Chen, J. I.; Ginger, D. S., Photoisomerization Quantum Yield of Azobenzene-Modified DNA Depends on Local Sequence. J Am Chem Soc 2013, 135, 8382-7.

3. Rau, H., Photoisomerization of Azobenzenes. In Photoreactive Organic Thin Films, Sekkat, Z.; Knoll, W., Eds. Academic Press: Amsterdam, 2002; pp 3-47.

4. MacKerell, A. D., Jr.; Banavali, N.; Foloppe, N., Development and Current Status of the Charmm Force Field for Nucleic Acids. Biopolymers 2000, 56, 257-65.

5. McCullagh, M.; Franco, I.; Ratner, M. A.; Schatz, G. C., DNA-Based Optomechanical Molecular Motor. J Am Chem Soc 2011, 133, 3452-9.

6. Mackerell, A. D.; Feig, M.; Brooks, C. L., Extending the Treatment of Backbone Energetics in Protein Force Fields: Limitations of Gas-Phase Quantum Mechanics in Reproducing Protein Conformational Distributions in Molecular Dynamics Simulations. J Comput Chem 2004, 25, 1400-1415.

7. Bayly, C. I.; Cieplak, P.; Cornell, W. D.; Kollman, P. A., A Well-Behaved Electrostatic Potential Based Method Using Charge Restraints for Deriving Atomic Charges - the Resp Model. J Phys Chem-Us 1993, 97, 10269-10280.

8. Case, D., et al., Amber 2015. University of California, San Francisco 2015.

9. Lu, X. J.; Olson, W. K., 3dna: A Versatile, Integrated Software System for the Analysis, Rebuilding and Visualization of Three-Dimensional Nucleic-Acid Structures. Nat Protoc 2008, 3, 1213-1227.

10. Humphrey, W.; Dalke, A.; Schulten, K., Vmd: Visual Molecular Dynamics. J Mol Graph Model 1996, 14, 33-38.

11. Jorgensen, W. L.; Chandrasekhar, J.; Madura, J. D.; Impey, R. W.; Klein, M. L., Comparison of Simple Potential Functions for Simulating Liquid Water. J Chem Phys 1983, 79, 926-935.

12. Pronk, S., et al., Gromacs 4.5: A High-Throughput and Highly Parallel Open Source Molecular Simulation Toolkit. Bioinformatics 2013, 29, 845-54.

13. Bussi, G.; Donadio, D.; Parrinello, M., Canonical Sampling through Velocity Rescaling. J Chem Phys 2007, 126.

14. Miyamoto, S.; Kollman, P. A., Settle - an Analytical Version of the Shake and Rattle Algorithm for Rigid Water Models. $J$ Comput Chem 1992, 13, 952-962.

15. Essmann, U.; Perera, L.; Berkowitz, M. L.; Darden, T.; Lee, H.; Pedersen, L. G., A Smooth Particle Mesh Ewald Method. J Chem Phys 1995, 103, 8577-8593.

16. Bonomi, M., et al., Plumed: A Portable Plugin for FreeEnergy Calculations with Molecular Dynamics. Comput Phys Commun 2009, 180, 1961-1972. 

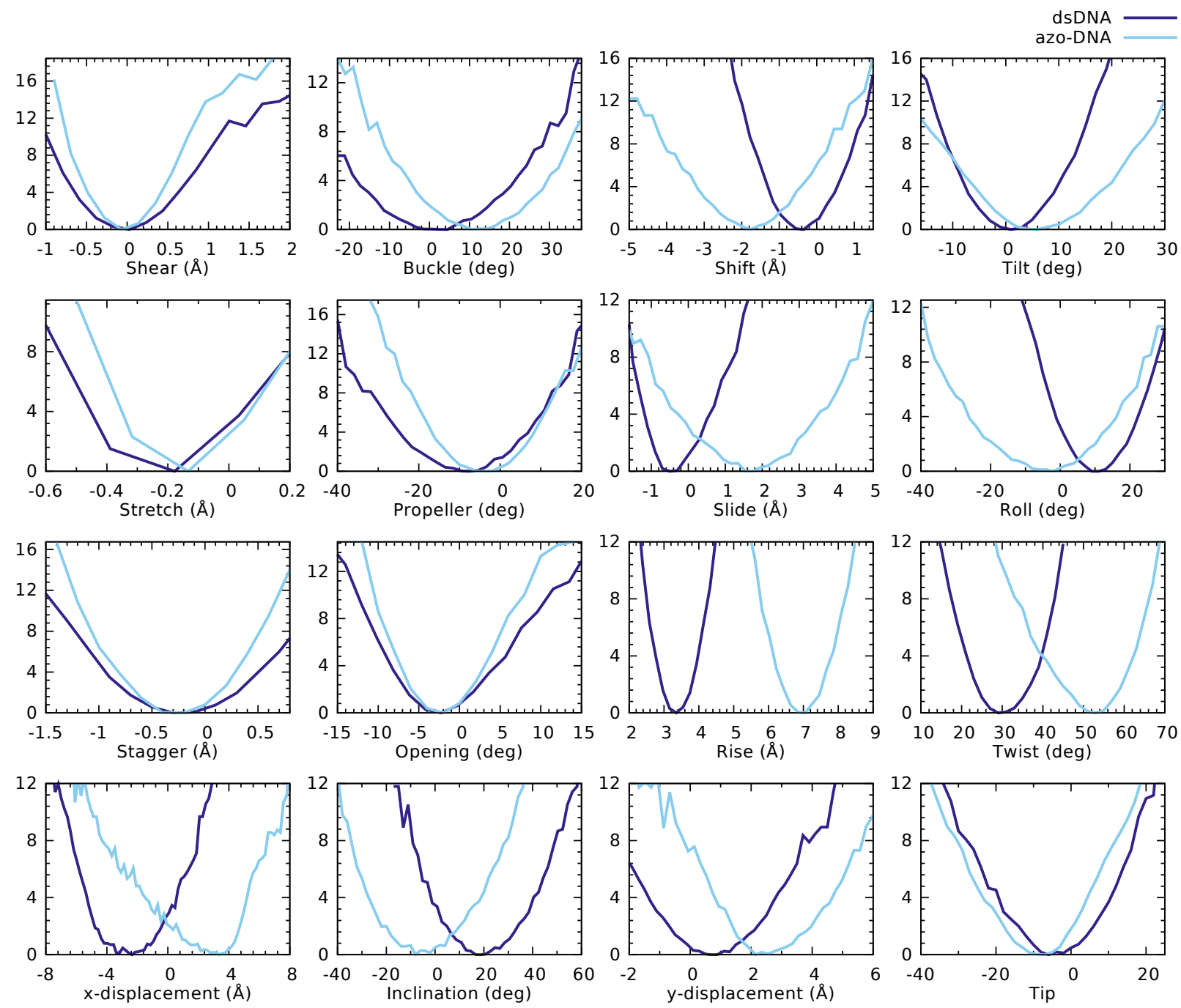

Supplementary Figure S1. Free energy surfaces of structural order parameters. Shown for comparison are non-modified double-stranded DNA (dsDNA) and azobenzene-modified DNA (azo-DNA), both fully matched. The bases considered for this analysis were those four bordering the azobenzene moiety. For the parameters shear, buckle, stretch, propeller, stagger, and opening, not much difference is seen between non-modified and azobenzene-containing DNA. Interestingly, though the average values of the parameters do not change much, the variance for the parameters is much lower (the free energy surface is narrower) for the azo-DNA as for the dsDNA. This would indicate that an azobenzene moiety inserted into DNA has the effect of stiffening the polymer. As expected, the step parameters which "step over" azobenzene are substantially different between azo-DNA and dsDNA. This is especially notable in the free energy profile of rise, whose average value of azo-DNA is almost twice that of dsDNA. 


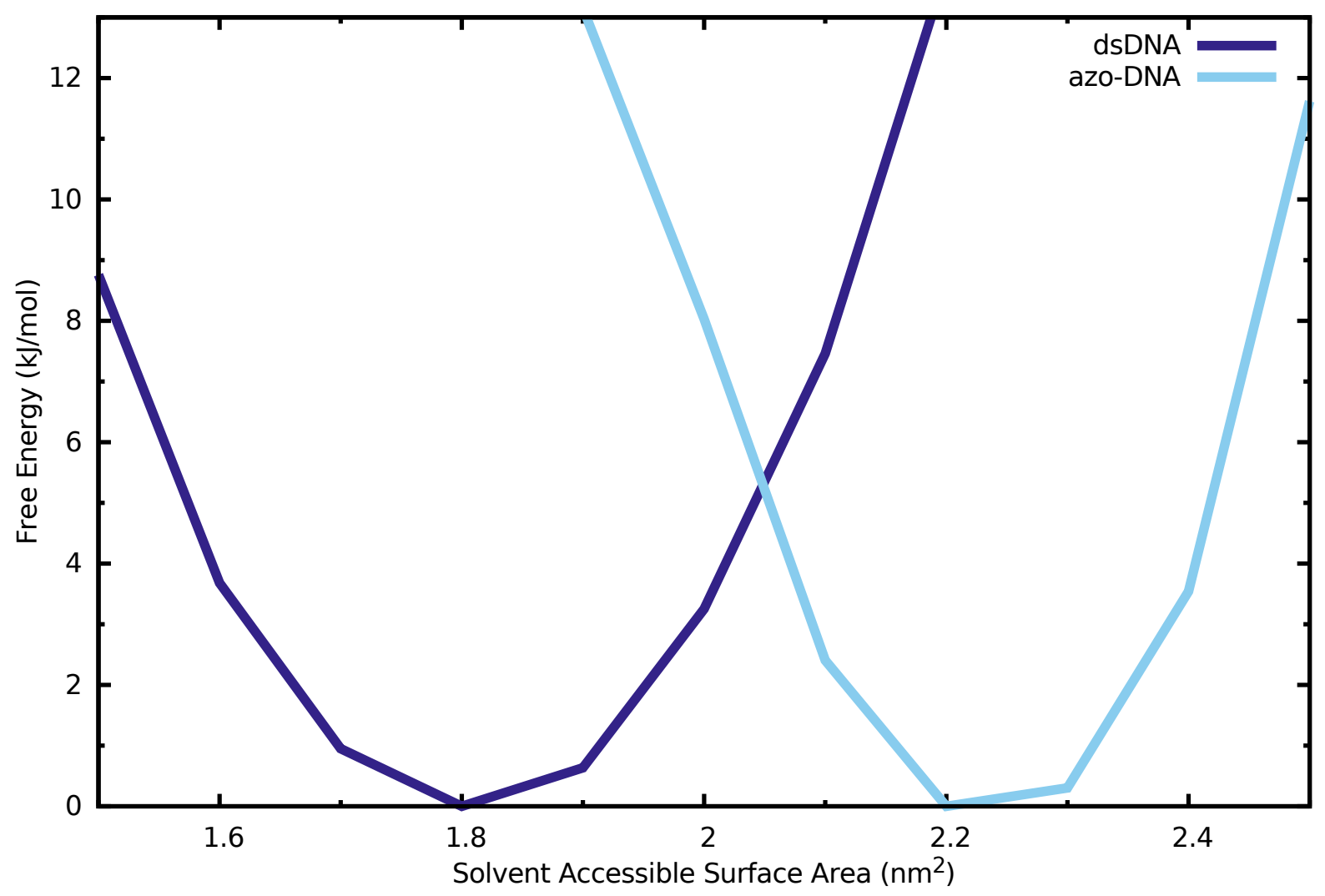

Supplementary Figure S2. Free energy comparison of double-stranded DNA (dsDNA) and azobenzene-modified DNA (azo-DNA) with regard to solvent accessible surface area (SASA) of the bases neighboring the azobenzene moiety. Only the heavy atoms of the sixmembered rings were considered. A slight elevation of SASA between azo-DNA and dsDNA indicates that the center of the DNA helix is slightly more exposed to the solvent when azobenzene is present, indicating imperfect stacking between azobenzene and its neighboring bases. The trans-azobenzene is slightly oriented toward the major groove of the DNA, though it still remains between and pi-stacks with its neighboring bases. 


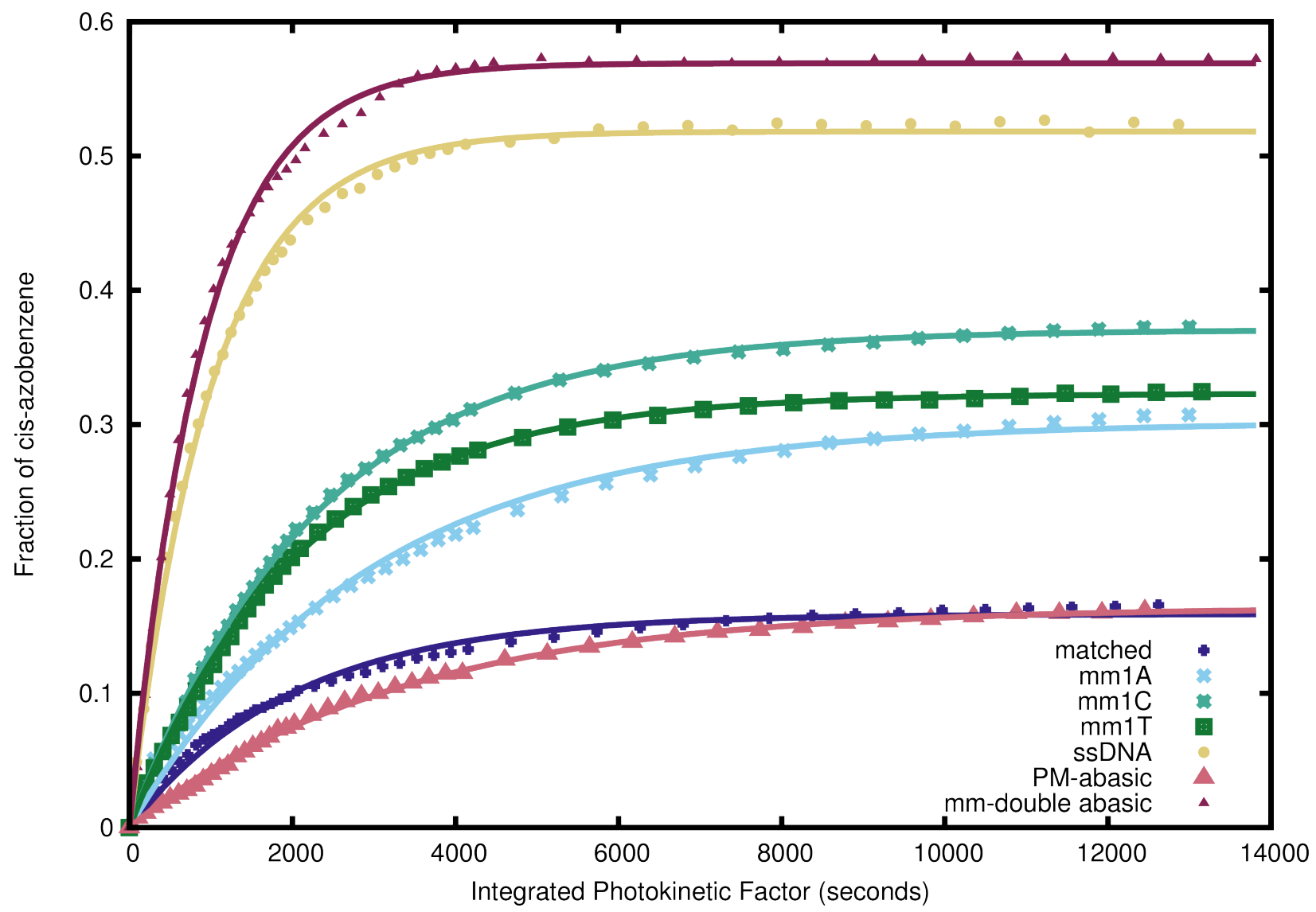

Supplementary Figure S3. Fraction of cis-azobenzene as a function of integrated photokinetic factor for matched, $\mathrm{mm} 1 \mathrm{~A}, \mathrm{~mm} 1 \mathrm{C}, \mathrm{mm} 1 \mathrm{~T}$, ssDNA, PM-abasic, and mm-double abasic sequences to extract the respective quantum yields. 


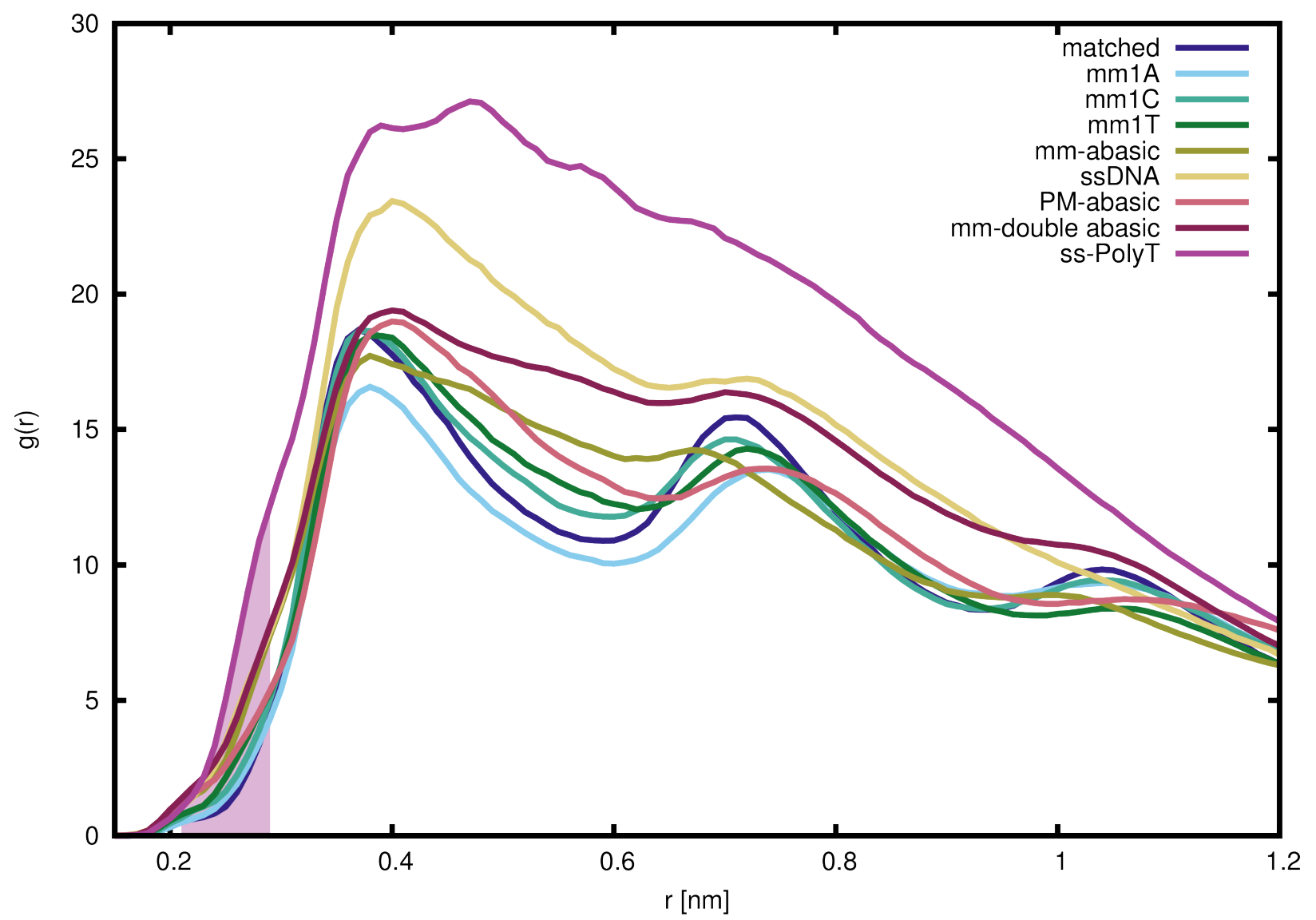

Supplementary Figure S4. Radial distribution function $g(r)$ between the outer phenyl ring of azobenzene and the remainder of the DNA. The first peak near $r=0.4 \mathrm{~nm}$ corresponds to the DNA bases neighboring the azobenzene. The shaded region illustrates the range of distances that contribute to calculation of the occupation number as outlined in the Methods section. 